\title{
Estudo da Reatividade Microvascular em Pacientes Hipertensos com Adiposidade Corporal Elevada
}

\author{
Microvascular Reactivity in Hypertensive Patients with High Body Adiposity \\ Jenifer d'El-Rei, ${ }^{1}$ Michelle Rabello Cunha, ${ }^{1}$ Samanta de Souza Mattos, ${ }^{1}$ Bianca Cristina Marques, ${ }^{1}$ Viviane Prangiel \\ de Menezes, ${ }^{1}$ Ana Rosa Cunha, ${ }^{1}$ Érica Monteiro França, ${ }^{1}$ Wille Oigman, ${ }^{1}$ Mario Fritsch Neves ${ }^{1}{ }^{[0}$ \\ Universidade do Estado do Rio de Janeiro - Clínica Médica, ${ }^{1}$ Rio de Janeiro, RJ - Brasil
}

\section{Resumo}

Fundamento: Diversos índices antropométricos têm sido propostos para determinar a associação entre excesso de peso e fatores de risco cardiovascular.

Objetivo: Avaliar a relação entre adiposidade corporal e reatividade microvascular em pacientes hipertensos sob terapia anti-hipertensiva.

Métodos: Pacientes hipertensos tratados de 40 a 70 anos foram submetidos à avaliação de índices antropométricos: conicidade (IC), adiposidade corporal (IAC), adiposidade visceral (IAV) e relação cintura-estatura (RCE). Os participantes foram divididos pelos tercis de percentual de gordura $(\% \mathrm{G})$ obtido pela bioimpedância elétrica (BIA) e submetidos a teste de reatividade microvascular (laser speckle contrast image), medida da velocidade da onda de pulso (VOP). O valor de $p<0,05$ foi considerado estatisticamente significativo.

Resultados: A variação da área sob a curva (ASC) da perfusão cutânea foi inferior no tercil superior (97 \pm 57\% vS. 67 $\pm 36 \%$; $p=0,027)$. O \%G apresentou correlação significativa com $\operatorname{RCE}(r=0,77 ; p<0,001), I A V(r=0,41 ; p=0,018), I C(r=0,60$; $p<0,001)$ e IAC $(r=0,65 ; p<0,001)$ nos homens e somente com $\operatorname{RCE}(r=0,55 ; p<0,001)$ e IAC $(r=0,60 ; p<0,001)$ nas mulheres. Na regressão linear, a ASC mostrou associação independente com o \%G $(\beta=-3,15 ; p=0,04)$ nas mulheres e com a glicemia $(\beta=-1,15 ; p=0,02)$ nos homens. Não houve diferença nas medidas de VOP.

Conclusão: Os índices antropométricos de obesidade foram mais associados ao \%G nos homens. A maior adiposidade corporal foi relacionada com menor reatividade microvascular, o que foi mais evidente nas mulheres. Não houve diferença na rigidez arterial, o que pode ter sido influenciado pelo tratamento anti-hipertensivo. (Arq Bras Cardiol. 2020; 115(5):896-904)

Palavras-chave: Hipertensão/efeitos do tratamento; Adiposidade; Endotélio; Permeabilidade Capilar.

\begin{abstract}
Background: Several anthropometric indexes have been proposed to determine the association between overweight and cardiovascular risk factors.

Objective: To evaluate the relationship between body adiposity and microvascular reactivity in hypertensive patients under antihypertensive therapy.
\end{abstract}

Methods: Treated hypertensive patients aged 40 to 70 were submitted to evaluation of anthropometric indexes: conicity (Cl), body adiposity (BAl), visceral adiposity (VAI) and waist-to-height ratio (WHtR). Participants were divided by the terciles of fat percentage (\%F) obtained by bioelectrical impedance. The patients underwent microvascular reactivity test (Laser Speckle Contrast Image) and pulse wave velocity (PWV) measurement. The $p$ value $<0.05$ was considered statistically significant.

Results: The variation of the area under the curve (AUC) of the skin perfusion was lower in the upper tercile $(97 \pm 57 \%$ vs. $67 \pm 36 \% ; p=0.027) . \% F$ showed significant correlation with WHtR $(r=0.77 ; p<0.001)$, VAl $(r=0.41 ; p=0.018), C l(r=0.60 ; p<0.001), B A l(r=0.65 ; p<0.001)$ in men and only with WHtR $(r=0.55 ; p<0.001)$ and BAI $(r=0.60 ; p<0.001)$ in women. In linear regression, AUC was independently associated with \% $(\beta=-3.15$; $p=0.04)$ in women and with blood glucose $(\beta=-1.15 ; p=0.02)$ in men. There was no difference in PWV measurements.

Conclusion: Anthropometric indices were more associated with \% in men. Higher body adiposity was associated with lower microvascular reactivity, which was more evident in women. There was no difference in arterial stiffness, which may have been influenced by antihypertensive treatment. (Arq Bras Cardiol. 2020; 115(5):896-904)

Keywords: Hypertension/drug effects; Adiposity; Endothelium; Capillary Permeability

Full texts in English - http://www.arquivosonline.com.br

Correspondência: Mario Fritsch Neves •

Universidade do Estado do Rio de Janeiro - Clínica Médica - Av. 28 de Setembro, 77. CEP 20551-030, Rio de Janeiro, RJ - Brasil

E-mail:mariofneves@gmail.com

Artigo recebido em 10/06/2019, revisado em 13/09/2019, aceito em 29/10/2019

DOI: https://doi.org/10.36660/abc.20190364 


\section{Introdução}

A Organização Mundial da Saúde (OMS) aponta a obesidade como um dos maiores problemas de saúde pública no mundo e, no Brasil, ela vem crescendo cada vez mais. Evidências apontam que mais de $50 \%$ da população apresentam-se na faixa de sobrepeso e obesidade. ${ }^{1,2}$

O diagnóstico correto de obesidade ou sobrepeso requer algumas formas de quantificação da composição corporal. As técnicas de imagem são alternativas que oferecem maior precisão; no entanto, a simplicidade de utilização destaca os métodos antropométricos como bons instrumentos para avaliação da gordura corporal. Diante disso, diversos índices antropométricos têm sido propostos para determinar a associação entre excesso de peso e fatores de risco cardiovascular. $^{3}$

O índice de massa corporal (IMC) é o que tem maior divulgação em estudos, mas não se correlaciona à distribuição da gordura corporal. Assim, as medidas da circunferência da cintura (CC) e a razão cintura-quadril (RCQ) são os indicadores mais utilizados na aferição da distribuição central do tecido adiposo. Outros que vêm demonstrando forte ligação com os fatores de risco cardiovascular são: o conicidade (IC), a relação cintura-estatura (RCE) e, mais recentemente, a adiposidade corporal (IAC) e o adiposidade visceral (IAV). ${ }^{3}$

$\mathrm{Na}$ obesidade, o tecido adiposo perivascular torna-se altamente inflamado e induz disfunção vascular por secreção aumentada de fatores de vasoconstrição, como os principais componentes do sistema renina-angiotensina e adipocinas pró-inflamatórias, os quais são importantes contribuintes para ativação endotelial e inflamação vascular. ${ }^{4}$

Na prática clínica, é essencial a identificação de parâmetros que possam refletir, de maneira mais precisa e viável, a distribuição do tecido adiposo (visceral ou subcutâneo) e sua relação com as alterações metabólicas e inflamatórias que levam ao prejuízo da saúde vascular e, consequentemente, aumentam o risco cardiovascular. Desse modo, este estudo teve como objetivo avaliar a relação entre adiposidade corporal e reatividade microvascular em pacientes hipertensos sob terapia anti-hipertensiva, além de correlacionar o percentual de gordura corporal e a influência do sexo aos índices antropométricos de adiposidade e risco cardiovascular.

\section{Métodos}

\section{População do estudo}

Pacientes hipertensos com idade entre 40 e 70 anos, de ambos os sexos, em uso de anti-hipertensivo por pelo menos quatro semanas, foram selecionados e admitidos em um estudo transversal. Os critérios de exclusão foram IMC $\geq 40$ $\mathrm{kg} / \mathrm{m}^{2}$, diabetes melito, terapia de reposição hormonal e uso de betabloqueador ou estatina. Para análise dos resultados, os pacientes foram divididos de acordo com o tercil do \%G, diferenciado pelo sexo. Nas mulheres, o ponto de corte dos tercis foi de 36,49 e $39,87 \%$, enquanto nos homens, foi de 25,27 e 28,95\%. O protocolo foi aprovado pelo Comitê de Ética local (50751314.9.0000.5259), e todos os participantes leram e assinaram o termo de consentimento livre e esclarecido em conformidade com o descrito na resolução 466/2012.

\section{Avaliação nutricional}

Seguindo as técnicas preconizadas pela OMS, o peso corporal foi aferido em balança digital da marca Filizola ${ }^{\circledR}$ com capacidade máxima de $180 \mathrm{~kg} .{ }^{5} \mathrm{Na}$ mesma balança foi verificada a estatura, a partir do antropômetro. O IMC foi calculado dividindo-se o peso corporal (em quilograma) pelo quadrado da altura (Alt; em metro). Os pontos de corte adotados para a classificação nutricional foram baseados nos critérios propostos pela OMS. ${ }^{6}$

As circunferências da cintura e do quadril foram obtidas com auxílio de fita métrica inextensível. A CC foi determinada no ponto médio entre a última costela e a crista ilíaca. A avaliação da circunferência do quadril (CQ) foi realizada no maior diâmetro da região glútea. A partir dessas medidas, foi calculada a RCQ. ${ }^{6}$

O cálculo da RCE foi feito conforme fórmula RCE $=\mathrm{CC} /$ Alt, e o cálculo do IC foi realizado utilizando a fórmula ${ }^{7} \mathrm{IC}=$ CC/0,109 $\times \sqrt{ }$ Peso/Alt.

O IAC foi calculado a partir da medida da CQ e da estatura: ${ }^{8}$ IAC $(\% \mathrm{G})=\mathrm{CQ} /($ Alt $\times$ VAlt $)-18$.

O IAV foi calculado levando em consideração as variações por gênero: ${ }^{9}$

- Homens: IAV $=(\mathrm{CC} / 39,68+1,88 \times \mathrm{IMC}) \times(\mathrm{TG} / 1,03) \times$ $(1,31 / \mathrm{HDL})$

- Mulheres: IAV $=(\mathrm{CC} / 39,68+1,89 \times \mathrm{IMC}) \times(\mathrm{TG} / 0,81) \times$ (1,52/HDL).

Em que TG = triglicerídeo $(\mathrm{mmol} / \mathrm{l}) ; \mathrm{HDL}=$ lipoproteina de alta densidade (do inglês, high density lipoprotein) (mmol/l).

A BIA foi realizada com o aparelho tetrapolar Biodynamics ${ }^{\circledR}$ modelo 310 e, utilizado para avaliação do \%G, seguindo recomendações prévias. ${ }^{10}$

\section{Avaliação laboratorial}

Amostras de sangue venoso foram coletadas após jejum de 8 horas. Glicose sérica, creatinina, colesterol total, HDL e TG foram medidos com uma técnica de autoanálise (Technicon DAX96, Miles Inc). A proteína C reativa (PCR) foi aferida pelo método de turbidimetria. A avaliação da função renal foi realizada utilizando a taxa de filtração glomerular (TFG), estimada pela equação Chronic Kidney Disease-Epidemiology Collaboration (CKD-EPI). ${ }^{11}$ A insulina foi medida por radioimunoensaio, e o índice de homeostatic model assessment-insulin resistance (HOMA-IR) = [glicemia de jejum $(\mathrm{mmol} / \mathrm{l}) \mathrm{x}$ insulina de jejum $(\mathrm{mUl} / \mathrm{ml}) / 22,5$ ] foi usado para estimar a sensibilidade à insulina. ${ }^{12}$

\section{Avaliação de pressão arterial e idades vascular e cardiometabólica}

As medidas da pressão arterial sistólica (PAS) e diastólica (PAD) foram obtidas com aparelho digital calibrado (modelo HEM-705CP, OMRON Healthcare Inc., Illinois), realizadas com o paciente em posição sentada e após cinco minutos de repouso. O cálculo de idade vascular foi baseado no Framingham Heart Study. ${ }^{13}$ 
A idade cardiometabólica foi obtida pelo endereço eletrônico https://myhealthcheckup.com, acessando o item Cardiometabolic Age. ${ }^{14} \mathrm{~A}$ síndrome metabólica foi definida de acordo com os critérios estabelecidos pelo Programa Nacional de Educação e Tratamento sobre Colesterol (NCEP ATP III). ${ }^{15}$

\section{Reatividade microvascular}

A reatividade microvascular foi avaliada utilizando o método laser speckle contrast image (LSCI) (Pericam PSI System, Perimed, Suécia), em combinação com a hiperemia reativa pós-oclusão (HRPO) para análise contínua das alterações de perfusão cutânea microvasculares dependentes de endotélio expressas em unidades de perfusão arbitrárias (UPA). Com essas análises, foram obtidos a média do período basal, a ASC do período basal de um minuto, a média do pico de HRPO e a ASC do período de um minuto após a oclusão. A condutância vascular cutânea (CVC) foi obtida pela fórmula: CVC = perfusão basal (ou HRPO)/pressão arterial média (PAM).

\section{Parâmetros hemodinâmicos centrais}

A análise da onda de pulso da artéria radial foi feita utilizando um dispositivo de tonometria disponível comercialmente (SphygmoCor; AtCor Medical, Sydney, Austrália). O aumento de pressão (AP) é a diferença entre a segunda e a primeira pressão de pico sistólico, e o augmentation index (Aix) é definido como a razão entre o AP e a pressão de pulso aórtica.

\section{Velocidade da onda de pulso}

As ondas de pulso foram obtidas transcutaneamente pelo aparelho COMPLIOR-SP (Alam Medical, France), por meio de transdutores colocados sobre a carótida direita e, ao mesmo tempo, sobre a artéria femoral direita. A distância entre os pulsos carotídeo e femoral foi medida diretamente com o auxílio de uma fita métrica inextensível, sendo o valor multiplicado por 0,8 para fins de cálculo da VOP. Essa medida foi corrigida calculando a VOP normalizada (VOP-CF-N), com a seguinte fórmula: VOP-CF-N $=(\mathrm{VOP}-\mathrm{CF} / \mathrm{PAM}) \times 100 .{ }^{16}$

\section{Análise estatística}

Os resultados foram expressos em média \pm desviopadrão. Para determinação do tamanho da amostra para este estudo, foi considerada a equivalência da variação da dilatação mediada por fluxo (DMF) observada em obesos. Desse modo, para uma diferença de 3,0\% na DMF, desviopadrão de 4,0\%, com $80 \%$ de poder de estudo e significância em 5\%, um número mínimo de 22 participantes em cada grupo seria necessário. Considerando uma perda estimada de $10 \%$ da amostra, a quantidade mínima foi definido

Tabela 1 - Parâmetros clínicos divididos pelos tercis do percentual de gordura

\begin{tabular}{|c|c|c|c|c|}
\hline \multirow[b]{2}{*}{ Parâmetros } & \multicolumn{3}{|c|}{ Percentual de gordura } & \multirow[b]{2}{*}{ Valor de $p$} \\
\hline & $\begin{array}{l}1^{0} \text { tercil } \\
(n=27)\end{array}$ & $\begin{array}{l}2^{\circ} \text { tercil } \\
(n=27)\end{array}$ & $\begin{array}{l}3^{\circ} \text { tercil } \\
(n=27)\end{array}$ & \\
\hline Idade (anos) & $57 \pm 6$ & $58 \pm 7$ & $60 \pm 7$ & 0,116 \\
\hline $\operatorname{RCV}(\%)$ & $15,6 \pm 10,5$ & $14,2 \pm 9,9$ & $20,8 \pm 12,9$ & 0,079 \\
\hline Idade vascular (anos) & $70 \pm 11$ & $68 \pm 12$ & $77 \pm 10^{*}+t$ & 0,007 \\
\hline Idade CM (anos) & $55 \pm 7$ & $55 \pm 8$ & $60 \pm 8^{*}$ & 0,025 \\
\hline PAS (mmHg) & $136 \pm 9$ & $135 \pm 13$ & $140 \pm 11$ & 0,173 \\
\hline $\mathrm{PAD}(\mathrm{mmHg})$ & $84 \pm 8$ & $81 \pm 10$ & $86 \pm 8$ & 0,137 \\
\hline Pressão de pulso (mmHg) & $52 \pm 6$ & $54 \pm 9$ & $54 \pm 8$ & 0,608 \\
\hline \multicolumn{5}{|l|}{ Estilo de vida, $\mathrm{n}(\%)$} \\
\hline Etilismo & $11(41)$ & $12(44)$ & $13(48)$ & 0,861 \\
\hline Sedentarismo & $22(82)$ & $19(70)$ & $22(82)$ & 0,526 \\
\hline \multicolumn{5}{|c|}{ Uso de anti-hipertensivo, $\mathrm{n}(\%)$} \\
\hline Diurético & $26(96)$ & $25(93)$ & $26(96)$ & 0,769 \\
\hline ISRA & $23(85)$ & $25(93)$ & $24(89)$ & 0,687 \\
\hline ACC & $8(30)$ & $5(19)$ & $5(19)$ & 0,526 \\
\hline Monoterapia & $4(15)$ & $2(7)$ & $4(15)$ & 0,493 \\
\hline Com dois fármacos & $16(59)$ & $22(82)$ & $18(67)$ & \\
\hline Com três fármacos & $7(26)$ & $3(11)$ & $5(19)$ & \\
\hline
\end{tabular}

Dwwados expressos em média \pm desvio-padrão ou em proporções, quando indicado. Valor de p corresponde ao Qui-quadrado para variáveis categóricas e One-Way Anova para variáveis numéricas com pós-teste de Tukey, sendo ${ }^{*} p<0,05$ vs. $1^{\circ}$ tercil, ${ }^{+\dagger} p<0,01$ vs. $2^{\circ}$ tercil. $R C V$ : risco cardiovascular; CM: cardiometabólica; PAS: pressão arterial sistólica; PAD: pressão arterial diastólica; ISRA: inibidor do sistema renina-angiotensina; ACC: antagonista do canal de cálcio. 
em 72 participantes. O teste de Shapiro-Wilk foi utilizado para avaliar distribuição normal. Os tercis do \%G foram comparados pelo teste One-Way ANOVA, seguido pelo pósteste de Tukey. As variáveis categóricas foram apresentadas como frequência e percentual e comparadas pelo teste do Qui-quadrado. O coeficiente de Pearson foi obtido em cada teste de correlação entre as variáveis contínuas. Foi considerado um intervalo de confiança de $95 \%$, sendo estatisticamente significativo quando $p<0,05$. A regressão linear foi realizada respeitando os pressupostos necessários, incluindo a ausência de multicolinearidade, considerando a ASC como variável dependente, ajustada por idade e por PAS, e realizada separadamente nos grupos de homens e mulheres. As análises estatísticas foram feitas pelo programa Statistical Package for the Social Sciences (SPSS) versão 20.0 para Windows (SPSS, Chicago, IL).

\section{Resultados}

Os resultados apresentados a seguir referem-se aos 81 pacientes que foram incluídos no estudo, com média de idade de $58 \pm 6$ anos, sendo $59 \%$ do sexo feminino $(n=48)$. A média do risco cardiovascular foi de $16,8 \pm 11,2 \%$, e a da pressão arterial, $138 \pm 11 / 83 \pm 9 \mathrm{mmHg}$. Os parâmetros clínicos da população dividida por tercis são encontrados na Tabela 1. Não houve diferença significativa na média de idade e risco cardiovascular entre os grupos. As idades vascular e cardiometabólica foram significativamente maiores no último tercil quando comparado ao primeiro.

$\mathrm{O}$ IMC foi bem maior no terceiro tercil, em relação ao primeiro e segundo. A CC foi maior no segundo e terceiro tercis, comparada à do primeiro, no sexo masculino, e no terceiro em relação ao primeiro no sexo feminino. A RCQ foi significativamente maior nas mulheres do terceiro tercil em relação ao primeiro, não sendo encontradas diferenças entre os homens. A RCE foi maior no último tercil quando comparado aos demais, tanto nos homens quanto nas mulheres.

O IC foi maior no último tercil no sexo masculino, quando comparado ao primeiro, e no último no sexo feminino quando comparado ao segundo e primeiro tercis. O IAC foi significativamente maior no terceiro tercil quando comparado aos outros dois. Já o IAV foi maior no terceiro tercil quando comparado ao segundo. O número de critérios para classificação da síndrome metabólica foi significativamente maior no último tercil em relação ao segundo (Tabela 2).

A Tabela 3 apresenta os dados laboratoriais sem diferença significativa em creatinina, perfis lipídico e glicêmico, PCR e TFG entre os grupos. O ácido úrico e a relação TG/HDL foram significativamente maiores no último tercil.

Na Tabela 4 encontram-se os dados dos testes vasculares. Nos parâmetros hemodinâmicos centrais, não foram encontradas diferenças significativas entre os grupos. A VOPCF e a VOP-CF-N não apresentaram diferenças estatísticas entre os tercis. Os dados obtidos pelo LSCI não demonstraram diferenças entre os grupos em perfusão, ASC e CVC basal e na HRPO. A variação da ASC foi significativamente menor no terceiro tercil quando comparado ao primeiro.

$\mathrm{O} \% \mathrm{G}$ exibiu correlações positivas e significativas com IMC, CC, RCE e índice de adiposidade, tanto nas mulheres quanto nos homens. Além desses resultados, entre os homens, a RCQ, o IC, o IAV e o número de critérios para síndrome metabólica também apresentaram correlação positiva e significativa com o \%G corporal (Tabela 5). A variação da ASC apresentou correlação negativa e significativa com o \%G corporal nas mulheres e com a glicemia entre os homens (Figura 1). Na regressão linear, essas associações permaneceram independentes, mesmo após ajustes para idade e PAS (Tabela 6).

\section{Discussão}

Este estudo avaliou a relação da adiposidade corporal com a reatividade microvascular e suas associações com diferentes índices antropométricos e metabólicos em uma população de hipertensos sob terapia medicamentosa. No que diz respeito aos parâmetros pressóricos, tanto periféricos quanto centrais, não foram encontradas diferenças entre os grupos, o que mostra que, do ponto de vista hemodinâmico, eles estavam bem equilibrados.

As idades vascular e cardiometabólica foram maiores no tercil superior de \%G, evidência de uma associação positiva entre acúmulo de gordura corporal e danos vasculares e metabólicos. Considerando que não houve diferença significativa no cálculo do risco cardiovascular, esse achado reforça a importância da estimativa desses parâmetros.

Valores superiores de ácido úrico e da relação TG/HDL foram encontrados no tercil de maior \%G, quando comparado com os tercis inferiores. A elevação do ácido úrico tem sido associada à síndrome metabólica. Estudos experimentais sugeriram que o ácido úrico pode penetrar nas fibras musculares lisas e vasculares, culminando no aumento da expressão de mediadores inflamatórios. As consequências são elevação da pressão arterial e hipertrofia de células musculares lisas vasculares. ${ }^{17-19} \mathrm{Um}$ estudo publicado recentemente, realizado na Índia com indivíduos adultos, demonstrou associação dos níveis de ácido úrico com parâmetros antropométricos de obesidade, como IMC, RCQ e RCE. ${ }^{18}$

A relação TG/HDL tem sido proposta como um simples marcador de resistência à insulina, funcionando como um biomarcador para identificar perfis de risco cardiometabólico. ${ }^{20}$ Pantoja-Torres et al. ${ }^{21}$ demonstraram, em uma população adulta eutrófica, associação positiva da relação TG/HDL com resistência insulínica. Essa relação também foi estudada por Baez-Duarte et al. ${ }^{22}$ mas em uma população adulta com IMC médio de $27,8 \mathrm{Kg} / \mathrm{m}^{2}$, na qual foi observada associação entre a relação TG/HDL e a menor sensibilidade à insulina e a presença de síndrome metabólica.

Uma vez que a disfunção endotelial é considerada um marcador do processo aterosclerótico, é crucial avaliar suas manifestações mais precoces em micro e macrocirculação. ${ }^{23} \mathrm{~A}$ avaliação da função endotelial pela reatividade microvascular, por meio do método $\mathrm{LSCl}$, não vem sendo utilizada nos ensaios clínicos com população obesa.

A reatividade microvascular na amostra estudada foi associada de modo negativo ao acúmulo de tecido adiposo, e a variação da ASC foi 30\% inferior no terceiro tercil, quando comparada à do primeiro. Suboc et al. ${ }^{24}$ demonstraram que a obesidade foi relacionada com pior função endotelial em 
Artigo Original

Tabela 2 - Parâmetros de adiposidade corporal divididos pelo tercil do percentual de gordura

\begin{tabular}{|c|c|c|c|c|}
\hline \multirow[b]{2}{*}{ Parâmetros } & \multicolumn{3}{|c|}{ Percentual de gordura } & \multirow[b]{2}{*}{ Valor de $p$} \\
\hline & $\begin{array}{l}1^{0} \text { tercil } \\
(n=27)\end{array}$ & $\begin{array}{l}2^{\circ} \text { tercil } \\
(n=27)\end{array}$ & $\begin{array}{l}3^{\circ} \text { tercil } \\
(n=27)\end{array}$ & \\
\hline IMC $\left(\mathrm{kg} / \mathrm{m}^{2}\right)$ & $26,1 \pm 3,7$ & $28,9 \pm 3,1$ & $31,4 \pm 2,8^{\prime * *+}$ & $<0,001$ \\
\hline \multicolumn{5}{|l|}{ Perímetro cintura $(\mathrm{cm})$} \\
\hline$\delta$ & $88,9 \pm 11,7$ & $98,8 \pm 6,6^{*}$ & $106,3 \pm 8,5^{\star * *}$ & $<0,001$ \\
\hline 운 & $86,5 \pm 8,3$ & $91,5 \pm 6,2$ & $97,7 \pm 8,8^{* * *}$ & $<0,001$ \\
\hline \multicolumn{5}{|l|}{$\mathrm{RCQ}$} \\
\hline$\hat{\sigma}$ & $0,88 \pm 0,08$ & $0,93 \pm 0,05$ & $0,95 \pm 0,04$ & 0,053 \\
\hline q & $0,80 \pm 0,05$ & $0,82 \pm 0,05$ & $0,86 \pm 0,06^{*}$ & 0,040 \\
\hline \multicolumn{5}{|l|}{ RCE } \\
\hline$\delta$ & $0,53 \pm 0,07$ & $0,56 \pm 0,03$ & $0,62 \pm 0,05^{\star \star *}+$ & 0,002 \\
\hline q & $0,54 \pm 0,05$ & $0,57 \pm 0,04$ & $0,63 \pm 0,05^{k *+1}$ & $<0,001$ \\
\hline \multicolumn{5}{|l|}{ Gordura corporal (\%) } \\
\hline$\delta$ & $20,0 \pm 4,6$ & $26,7 \pm 1,1^{+* n}$ & $31,8 \pm 2,5^{4 * t+t}$ & $<0,001$ \\
\hline q & $31,0 \pm 4,5$ & $38,5 \pm 1,0^{* *}$ & $44,6 \pm 8,4^{m+x+t}$ & $<0,001$ \\
\hline \multicolumn{5}{|l|}{ Índice de conicidade } \\
\hline$\hat{\sigma}$ & $1,25 \pm 0,87$ & $1,30 \pm 0,57$ & $1,33 \pm 0,62^{\star}$ & 0,026 \\
\hline o & $1,21 \pm 0,76$ & $1,21 \pm 0,53$ & $1,28 \pm 0,80^{*}+$ & 0,009 \\
\hline \multicolumn{5}{|l|}{ Índice de adiposidade corporal } \\
\hline 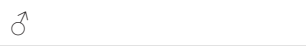 & $28,0 \pm 3,7$ & $27,4 \pm 1,4$ & $31,9 \pm 3,7^{\star+十}$ & 0,004 \\
\hline q & $34,3 \pm 3,4$ & $36,4 \pm 3,2$ & $40,9 \pm 4,6^{m+x+t}$ & $<0,001$ \\
\hline Índice de adiposidade visceral & $2,88 \pm 1,13$ & $2,51 \pm 1,04$ & $3,55 \pm 1,98^{\dagger}$ & 0,037 \\
\hline Critérios SM & $2,3 \pm 1,1$ & $2,1 \pm 0,9$ & $2,9 \pm 0,9^{\dagger}$ & 0,018 \\
\hline
\end{tabular}

Tabela 3 - Parâmetros laboratoriais divididos pelo tercil do percentual de gordura

\begin{tabular}{|c|c|c|c|c|}
\hline \multirow[t]{2}{*}{ Parâmetros } & \multicolumn{3}{|c|}{ Percentual de gordura } & \multirow{2}{*}{ Valor de $p$} \\
\hline & $\begin{array}{l}1^{0} \text { tercil } \\
(n=27)\end{array}$ & $\begin{array}{l}2^{\circ} \text { tercil } \\
(n=27)\end{array}$ & $\begin{array}{l}3^{\circ} \text { tercil } \\
(n=27)\end{array}$ & \\
\hline Creatinina (mg/dl) & $0,88 \pm 0,20$ & $0,89 \pm 0,20$ & $0,92 \pm 0,25$ & 0,851 \\
\hline Ácido úrico (mg/dl) & $5,29 \pm 1,60$ & $5,52 \pm 1,49$ & $6,40 \pm 1,69^{*}$ & 0,029 \\
\hline Colesterol total (mg/dl) & $209 \pm 47$ & $203 \pm 28$ & $216 \pm 36$ & 0,485 \\
\hline $\mathrm{HDL}$ (mg/dl) & $56 \pm 16$ & $61 \pm 20$ & $51 \pm 19$ & 0,164 \\
\hline LDL (mg/dl) & $126 \pm 38$ & $123 \pm 43$ & $129 \pm 34$ & 0,822 \\
\hline TG (mg/dl) & $131 \pm 49$ & $111 \pm 47$ & $130 \pm 58$ & 0,061 \\
\hline TG/HDL & $2,60 \pm 1,43$ & $2,28 \pm 1,92$ & $3,59 \pm 2,85^{\dagger}$ & 0,050 \\
\hline Glicose (mg/dl) & $94 \pm 11$ & $93 \pm 10$ & $96 \pm 11$ & 0,670 \\
\hline HOMA-IR & $3,38 \pm 1,69$ & $3,20 \pm 1,29$ & $3,91 \pm 1,89$ & 0,270 \\
\hline PCR-us (mg/dl) & $0,71 \pm 0,50$ & $0,73 \pm 0,49$ & $0,83 \pm 0,59$ & 0,655 \\
\hline TFG $\left(\mathrm{ml} / \mathrm{min} / 1,73 \mathrm{~m}^{2}\right)$ & $87 \pm 13$ & $84 \pm 19$ & $79 \pm 19$ & 0,544 \\
\hline
\end{tabular}

Dados expressos em média \pm desvio-padrão. Valor de $P$ corresponde ao Qui-quadrado para variáveis categóricas e One-Way Anova para variáveis numéricas com pós-teste de Tukey, sendo * $p<0,05$ vs. $1^{0}$ tercil; ${ }^{\dagger} p<0,05$ vs. $2^{\circ}$ tercil. HDL: lipoproteina de alta densidade; LDL, lipoproteína de baixa densidade; TG, triglicerídeo; HOMA-IR, homeostatic model assessment-insulin resistance; PCR-us, proteína C reativa ultrassensivel; TFG, taxa de filtração glomerular. 


\section{Artigo Original}

Tabela 4 - Parâmetros vasculares divididos pelo tercil do percentual de gordura

\begin{tabular}{|c|c|c|c|c|}
\hline \multirow[b]{2}{*}{ Parâmetros } & \multicolumn{4}{|c|}{ Percentual de gordura } \\
\hline & $\begin{array}{l}1^{0} \text { tercil } \\
(n=27)\end{array}$ & $\begin{array}{l}2^{\circ} \text { tercil } \\
(n=27)\end{array}$ & $\begin{array}{l}3^{\circ} \text { tercil } \\
(n=27)\end{array}$ & Valor de $p$ \\
\hline & \multicolumn{4}{|c|}{$\begin{array}{l}\text { Hemodinâmica central } \\
\text { Tonometria de aplanação }\end{array}$} \\
\hline PAS aórtica (mmHg) & $131 \pm 15$ & $130 \pm 17$ & $132 \pm 16$ & 0,855 \\
\hline PP aórtica (mmHg) & $46 \pm 11$ & $47 \pm 10$ & $46 \pm 12$ & 0,941 \\
\hline $\mathrm{AP}(\mathrm{mmHg})$ & $17 \pm 10$ & $16 \pm 7$ & $15 \pm 7$ & 0,746 \\
\hline Aix (\%) & $33 \pm 11$ & $32 \pm 10$ & $32 \pm 10$ & 0,945 \\
\hline Aix @ FC $75(\%)$ & $27 \pm 9$ & $27 \pm 9$ & $27 \pm 9$ & 0,997 \\
\hline $\mathrm{DE}(\%)$ & $34 \pm 4$ & $35 \pm 3$ & $35 \pm 4$ & 0,723 \\
\hline \multirow[t]{2}{*}{ SEVR } & $167 \pm 37$ & $156 \pm 27$ & $155 \pm 26$ & 0,272 \\
\hline & \multicolumn{4}{|c|}{ VOP } \\
\hline VOP-CF (m/s) & $10,0 \pm 1,8$ & $9,7 \pm 1,9$ & $9,7 \pm 2,0$ & 0,803 \\
\hline \multirow[t]{2}{*}{ VOP-CF-N (m/s) } & $9,9 \pm 1,7$ & $9,8 \pm 1,7$ & $9,4 \pm 1,9$ & 0,468 \\
\hline & \multicolumn{4}{|c|}{ LSCl } \\
\hline Perfusão basal (UAP) & $28,4 \pm 11,0$ & $32,2 \pm 10,7$ & $30,9 \pm 9,8$ & 0,410 \\
\hline Basal CVC (UPA/mmHg) & $0,28 \pm 0,11$ & $0,33 \pm 0,12$ & $0,30 \pm 0,09$ & 0,303 \\
\hline Perfusão HRPO (UPA) & $84,2 \pm 26,2$ & $87,2 \pm 22,2$ & $90,6 \pm 21,0$ & 0,601 \\
\hline HRPO CVC (UPA/mmHg) & $0,84 \pm 0,28$ & $0,89 \pm 0,25$ & $0,87 \pm 0,20$ & 0,743 \\
\hline ASC basal (UPA) & $1800 \pm 679$ & $2001 \pm 663$ & $1996 \pm 579$ & 0,334 \\
\hline ASC HRPO (UPA) & $3360 \pm 1190$ & $3257 \pm 856$ & $3261 \pm 882$ & 0,978 \\
\hline Variação de ASC (\%) & $97 \pm 57$ & $70 \pm 35$ & $67 \pm 36^{\circ}$ & 0,027 \\
\hline Variação de CVC (\%) & $218 \pm 105$ & $185 \pm 73$ & $211 \pm 90$ & 0,366 \\
\hline
\end{tabular}

Dados expressos em média \pm desvio-padrão. Valor de $p$ corresponde ao Qui-quadrado para variáveis categóricas e One-Way Anova para variáveis numéricas com pós-teste de Tukey, sendo * $p<0,05$ vs. $1^{\circ}$ tercil. PAS: pressão arterial sistólica; PP: pressão de pulso; AP: aumento de pressão; Aix: augmentation index; DE: duração de ejeção; SEVR: razão de viabilidade subendocárdica; VOP: velocidade da onda de pulso; CF: carótida femoral; CF-N: carótida femoral normalizada; LSIC: laser speckle de imagem contrastada; UPA: unidade de perfusão arbitrária; CVC: condutância vascular cutânea; HRPO: hiperemia reativa pós-oclusão; ASC: área sob a curva.

Tabela 5 - Correlação de Pearson dos índices de adiposidade e de risco cardiovascular com o percentual de gordura, por sexo

\begin{tabular}{|c|c|c|c|c|}
\hline \multirow{2}{*}{ Variáveis } & \multicolumn{2}{|c|}{ Mulheres $(n=48)$} & \multicolumn{2}{|c|}{ Homens $(n=33)$} \\
\hline & Coeficiente $r$ & Valor de $p$ & Coeficiente $r$ & Valor de $p$ \\
\hline IMC $\left(\mathrm{kg} / \mathrm{m}^{2}\right)$ & 0,556 & $<0,001$ & 0,738 & $<0,001$ \\
\hline $\mathrm{CC}(\mathrm{cm})$ & 0,476 & 0,001 & 0,767 & $<0,001$ \\
\hline $\mathrm{RCQ}$ & 0,215 & 0,152 & 0,505 & 0,003 \\
\hline RCE & 0,550 & $<0,001$ & 0,767 & $<0,001$ \\
\hline Índice de adiposidade corporal & 0,599 & $<0,001$ & 0,653 & $<0,001$ \\
\hline Índice de conicidade & 0,264 & 0,076 & 0,597 & $<0,001$ \\
\hline Índice de adiposidade visceral & $-0,037$ & 0,809 & 0,410 & 0,018 \\
\hline IV Framingham (anos) & 0,062 & 0,682 & $-0,005$ & 0,976 \\
\hline Idade CM (anos) & 0,242 & 0,109 & $-0,044$ & 0,810 \\
\hline Critérios SM & $-0,066$ & 0,662 & 0,464 & 0,007 \\
\hline
\end{tabular}

Correlação de Pearson. IMC: índice de massa corporal; CC: circunferência da cintura; RCQ: razão cintura-quadril; RCE: relação cintura-estatura; IV: idade vascular; CM:cardiometabólica; SM: síndrome metabólica. 

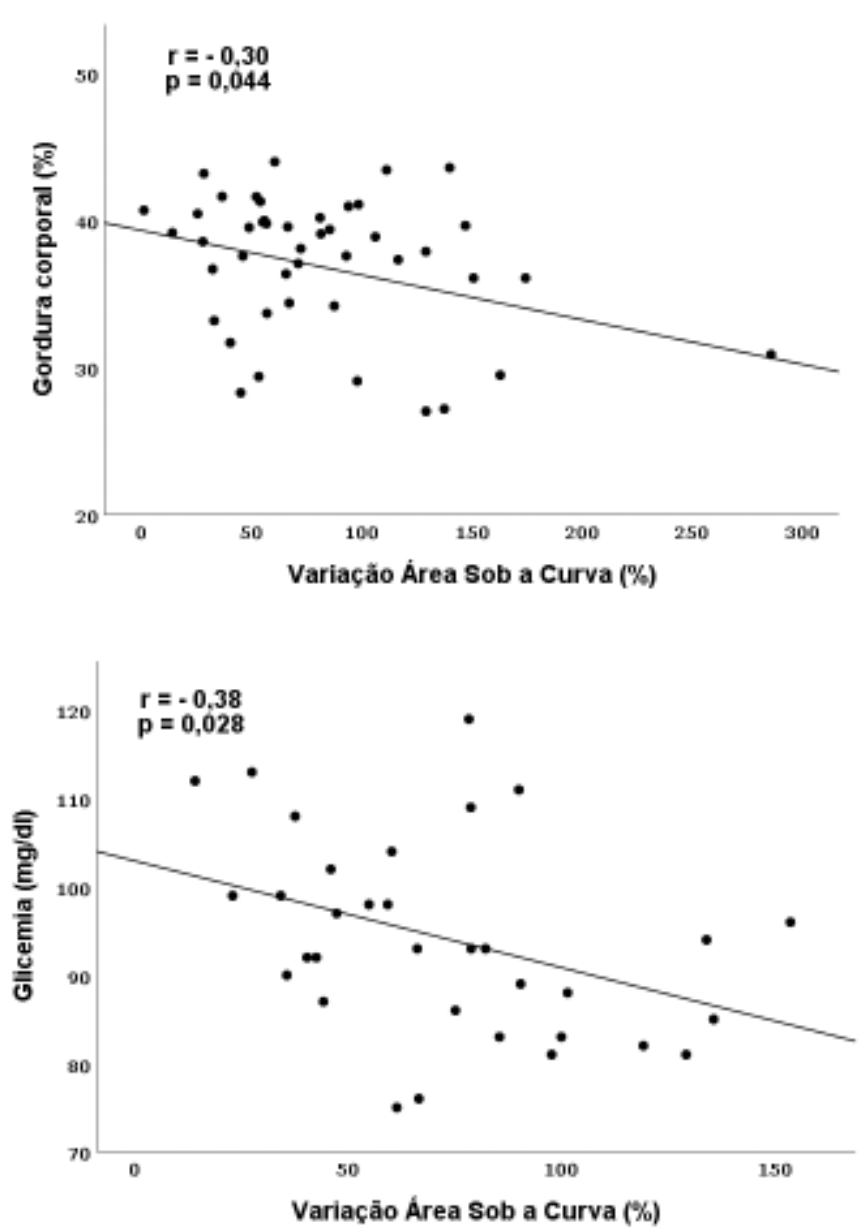

Figura 1 - Correlação da variação da área sob a curva da perfusão cutânea pelo método laser speckle contrast image com gordura corporal em mulheres e com glicemia em homens.

Tabela 6 - Regressão linear da variável dependente da variação da área sob a curva da perfusão cutânea pelo método laser speckle contrast image com gordura corporal no sexo feminino e com glicemia no sexo masculino, após ajuste para idade e pressão arterial sistólica.

\begin{tabular}{|c|c|c|c|c|c|c|}
\hline \multicolumn{2}{|c|}{ Variáveis dependentes } & \multirow{2}{*}{$\frac{\text { Variáveis independentes }}{\text { Gordura corporal (\%) }}$} & \multirow{2}{*}{$\begin{array}{c}\text { Coeficiente não padronizado } B \\
-3,15\end{array}$} & \multirow{2}{*}{$\begin{array}{l}\text { IC } 95 \% \\
-6,29 \\
-0,10\end{array}$} & \multirow{2}{*}{$\begin{array}{c}\text { Coeficiente padronizado Beta } \\
-0,32\end{array}$} & \multirow{2}{*}{$\begin{array}{c}\text { Valor de } p \\
0,049\end{array}$} \\
\hline ASC (\%) & 우 & & & & & \\
\hline & $\sigma^{\lambda}$ & Glicemia (mg/dl) & $-1,35$ & $\begin{array}{l}-2,47 \\
-0,22\end{array}$ & $-0,43$ & 0,020 \\
\hline
\end{tabular}

ASC: área sob a curva; đ̊: sexo masculino; ㅇ: sexo feminino.

adultos não diabéticos. Esses achados só se tornaram significativos quando os grupos foram divididos por sexo. A função endotelial, avaliada neste estudo pela variação da ASC, correlacionou-se à glicemia no sexo masculino, sugerindo uma possível associação importante entre função endotelial e sensibilidade à insulina nos homens. Já nas mulheres, essa correlação mostrou-se mais evidente com o \%G corporal, indicando uma provável relação direta entre tecido adiposo e função vascular no sexo feminino. Essas correlações se mantiveram após ajustes para a idade e para PAS, importantes fatores na regulação da função endotelial.

Com relação aos parâmetros de rigidez arterial, não foram encontradas diferenças entre os grupos estudados. 
Desamericq et al. ${ }^{25}$ não encontraram relação entre obesidade e aumento de rigidez arterial em uma amostra de adultos com fatores de risco cardiovascular associados, como diabetes tipo 2. Menezes et al. ${ }^{26}$ também não observaram associação entre obesidade e resistência insulínica com alteração vascular, tanto na função endotelial quanto na rigidez arterial. No estudo, a ligação da adiposidade com a rigidez arterial pode ter sido atenuada pela administração de fármacos vasoprotetores no esquema terapêutico dos pacientes, como os inibidores do sistema renina-angiotensina (ISRA).

O \%G obtido pela BIA no sexo masculino mostrou uma boa relação com todos os índices antropométricos de avaliação de adiposidade corporal e com o número de critérios para síndrome metabólica. Nas mulheres, tal correlação permaneceu somente para IMC, CC, RCE e IAC. Em 2007, um estudo realizado no Brasil, com o objetivo de determinar a relação entre os diversos indicadores de obesidade e o risco coronariano, mostrou que os indicadores apresentaram forte associação com RCE, destacando-se entre os homens o RCQ e o IC, enquanto para as mulheres entre 50 e 74 anos o IC foi o melhor marcador. Essa diferença pode ser explicada pela menopausa, fase em que ocorre perda da proteção fornecida pelo hormônio estrogênio, levando ao maior acúmulo de gordura abdominal, que contribui para a ocorrência de problemas cardiovasculares. ${ }^{27}$

Algumas limitações foram identificadas no estudo. A ausência de marcadores inflamatórios e adipocinas prejudicou a análise da inflamação como mecanismo da disfunção endotelial associada à adiposidade corporal aumentada. A PCR foi o único marcador avaliado e não se mostrou diferente entre os grupos estudados. Os efeitos de alguns anti-hipertensivos podem ter influenciado esse resultado. Nenhum método de imagem foi utilizado para quantificar o tecido adiposo visceral. Entretanto, o objetivo principal do trabalho foi utilizar métodos mais simples de avaliação da adiposidade corporal e sua associação com as alterações vasculares que podem caracterizar maior risco cardiovascular.

\section{Referências}

1. Associação Brasileira para o Estudo da Obesidade e da Síndrome Metabólica. Diretrizes brasileiras de obesidade. 4a.ed. São Paulo: Abeso; 2016. [acesso em 03 Jun. 2019]. Disponível em: https://abeso.org.br/wpcontent/uploads/2019/12/Diretrizes-Download-Diretrizes-Brasileiras-deObesidade-2016.pdf. .

2. Malta D, Bernal RTI, Lima MG, Araújo SSC, Silva MMA, Freitas MIF, et al. . Noncommunicable diseases and the use of health services: analysis of the National Health Survey in Brazil. Rev Saúde Pública. 2017; 51(Supl 1):110.

3. Haun DR, Pitanga FJG, lessa I. Waist/height ratio compared with other anthropometric indicators of obesity as a predictor of high coronary risk. Rev Assoc Med Bras. 2010;55(6):705-11.

4. Gu P, Xu A. Interplay between adipose tissue and blood vessels in obesity and vascular dysfunction. Rev Endocr Metab Disord. 2013;14(1):49-58.

5. World Health Organization. Physical status: the use and interpretation of anthropometry. Report of a WHO Expert Committee. Technical Report Series

\section{Conclusão}

$\mathrm{Na}$ amostra apresentada de pacientes hipertensos em tratamento, os índices antropométricos de obesidade foram mais associados ao \%G corporal entre os homens. O maior risco cardiovascular entre aqueles com maior adiposidade corporal foi mais evidenciado pelas maiores idades vascular e cardiometabólica nesse grupo de pacientes. A maior adiposidade corporal foi relacionada com menor reatividade microvascular, o que foi mais evidente entre as mulheres. Não houve diferença em relação à rigidez arterial, o que pode ser atribuído ao uso das medicações anti-hipertensivas que mantiveram níveis semelhantes de pressão arterial nos grupos estudados.

\section{Contribuição dos autores}

Concepção e desenho da pesquisa: d’El-Rei J, Oigman W, Neves MF; Obtenção de dados: d'El-Rei J, Cunha MR, Mattos SS, Marques BC, Menezes VP, Cunha AR, França EM; Análise e interpretação dos dados: d'El-Rei J, Cunha MR, Mattos SS, Marques BC, Menezes VP, Cunha AR, França EM, Oigman W, Neves MF; Análise estatística e Redação do manuscrito: d'El-Rei J, Cunha MR, Neves MF; Obtenção de financiamento: Neves MF; Revisão crítica do manuscrito quanto ao conteúdo intelectual importante: d'El-Rei J, Cunha MR, Mattos SS, Marques BC, Menezes VP, Cunha AR, Oigman W, Neves MF.

\section{Potencial conflito de interesses}

Declaro não haver conflito de interesses pertinentes.

\section{Fontes de financiamento}

O presente estudo foi parcialmente financiado pelo $\mathrm{CNPq}$ e FAPERJ.

\section{Vinculação acadêmica}

Este artigo é parte de tese de Doutorado de Jenifer d’El-Rei pela Universidade do Estado do Rio de Janeiro.

n. 854. Geneva: WHO; 1995. [acesso em 03 Jun. 2020]. Disponível em: http://www.who.int/childgrowth/publications/physical_status/en/index.html.

6. World Health Organization. Obesity: preventing and managing the global epidemic - report on a WHO consultation (WHO Technical Report Series 894). Geneva:: WHO; 2000.

7. Valdez R. A simple model-based index of abdominal adiposity. J Clin Epidemiol. 1991;44(9):955-6.

8. Bergman RN, Stefanovski D, Buchanan TA, Sumner AE, Reynolds JC, Sebring NG, et al. A better index of body adiposity. Obesity. 2011;19(5):1083-9.

9. Amato MC, Giordano C, Galia M, Criscimanna A, Vitabile S, Midiri M, et al. Visceral adiposity index: a reliable indicator of visceral fat function associated with cardiometabolic risk. Diabetes Care. 2010;33(4):920-2.

10. Lukaski HC, Bolonchuk WW, Hall CB, Siders WA. Validation of tetrapolar bioelectrical impedance method to assess human body composition. J Appl Physiol. 1986;60(4):1327-32. 
11. Levey AS, Stevens LA, Schmid CH, Zhang YL, Castro 3rd AF, Feldman HI, etal. A new equation to estimate glomerular filtration rate. 2009;150(9):604-12.

12. Matthews DR, Hosker JP, Rudenski S, Naylor BA, Treacher DF, Turner RC. Homeostasis model assessment: insulin resistance and beta-cell function from fasting plasma glucose and insulin concentrations in man. Diabetologia. 1985;28(7):412-9.

13. D'Agostino RB, Vasan RS, Pencina MJ, Wolf PA, Cobain M, Massaro JM, et al General cardiovascular risk profile for use in primary care: the Framingham Heart Study. Circulation. 2008;117(6):743-53.

14. Yang Q, Zhong Y, Ritchey M, Cobain M, Gillespie C, MerrittR, etal. Vital signs: predicted heart age and racial disparities in heart age among U.S. adults at the state level. 2015;64(34):950-8.

15. National Cholesterol Education Program (NCEP) Expert Panel on Detection, Evaluation, and Treatment of High Blood Cholesterol in Adults (Adult Treatment Panel III). Third Report of the National Cholesterol Education Program (NCEP) Expert Panel on Detection, Evaluation, and Treatment of High Blood Cholesterol in Adults (Adult Treatment Panel III) final report. Circulation. 2002;106(25):3143-421.

16. Scuteri A, Maria A, Gianni W, Assisi A, Volpe M. Arterial stiffness is an independent risk factor for cognitive impairment in the elderly: a pilot study. J Hypertens. 2005;23(6):1211-6.

17. Kanbay M, Segal M, Afsar B, Kang DH, Rodriguez-Iturbe B, Johnson RJ. The role of uric acid in the pathogenesis of human cardiovascular disease. Heart. 2013;99(11):759-66.

18. Mukhopadhyay P, Ghosh S, PanditK, Chatterjee P, Majhi B, Chowdhury S. Uric acid and its correlation with various metabolic parameters: a population-based study. Indian J Endocrinol Metab. 2019;23(1):134-9.

19. Gonçalves JP, Oliveira A, Severo M, Santos AC, Lopes C. Cross-sectional and longitudinal associations between serum uric acid and metabolic syndrome. Endocrine. 2012;41(3):450-7.
20. Jeppesen J, Hein HO, Suadicani P, Gyntelberg F. Relation of high TGlow HDL cholesterol and LDL cholesterol to the incidence of ischemic heart disease. An 8-year follow-up in the Copenhagen Male Study. Arterioscler Thromb Vasc Biol. 1997;17(6):1114-20.

21. Pantoja-Torres B, Toro-Huamanchumo CJ, Urrunaga-Pastor D Guarnizo-Poma M, Lazaro-Alcantara H Paico-Palacios S, et al. High triglycerides to $\mathrm{HDL}$-cholesterol ratio is associated with insulin resistance in normal-weight healthy adults. Diabetes Metab Syndr. 2019:13(1):382-8

22. Baez-Duarte BG, Zamora-Gínez I, González-Duarte R, Torres-Rasgado E, Ruiz-Vivanco G, Pérez-Fuentes R, et al. Triglyceride/high-density lipoprotein cholesterol (TG/HDL-C) index as a reference criterion of risk for metabolic syndrome (MetS) and low insulin sensitivity in apparently healthy subjects. Gac Med Mex. 2017;153(2):152-8.

23. Maranhão PA, Souza MGC, Panazzolo DG, Nogueira Neto JF, Bouskela E, Kraemer-Aguiar LG, et al. Metabolic changes induced by high-fat meal evoke different microvascular responses in accordance with adiposity status. Biomed Res Int. 2018;2018:5046508.

24. Suboc TMB, Dharmashankar K, Wang J, Ying R, Couillard AB, Tanner $M J$, et al. Moderate obesity and endothelial dysfunction in humans: influence of gender and systemic inflammation. Physiol Rep. $2013 ; 1(3): 1-10$

25. Desamericq G, Tissot CM, Akakpo S, Tropeano AI, Milasseau S, Macquin-Mavier I, et al. Carotid-femoral pulse wave velocity is not increased in obesity. Am J Hypertens. 2015;28(4):546-51.

26. Menezes VP, Cohen C, Del-Rei J, Oigman W, Neves MF, Medeiros FJ. Evaluation of endothelial function and arterial stiffness in obese individuals with insulin resistance. Nutr Health. 2019;25(2):85-92.

27. Pitanga FJG, Lessa I. Association of anthropometric indicators of obesity with coronary risk in adults in the city of Salvador, Bahia, Brazil. Rev Bras Epidemiol. 2007;10(2):239-48. 